\title{
200-DV-1OU Sediment and Pore Water Analysis and Report for Samples at Borehole C8096
}

Michael Lindberg

October 2011

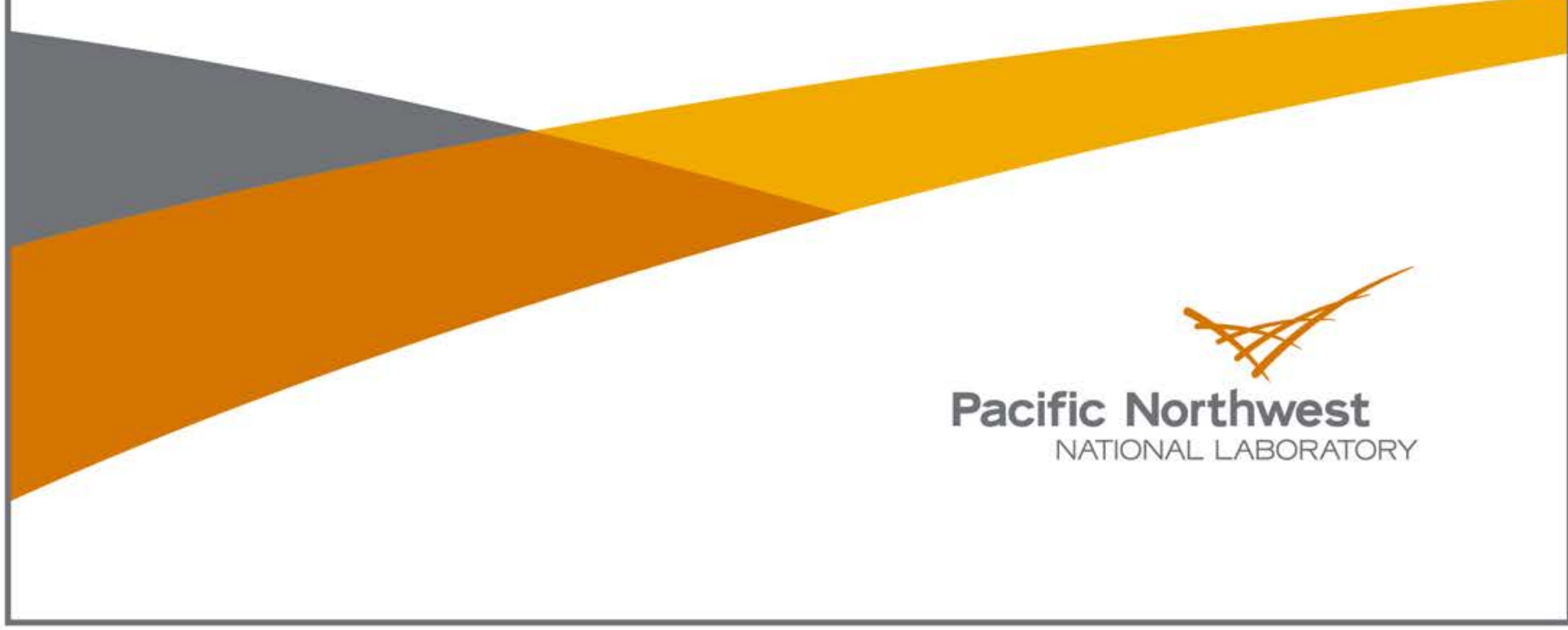




\title{
DISCLAIMER
}

This report was prepared as an account of work sponsored by an agency of the United States Government. Neither the United States Government nor any agency thereof, nor Battelle Memorial Institute, nor any of their employees, makes any warranty, express or implied, or assumes any legal liability or responsibility for the accuracy, completeness, or usefulness of any information, apparatus, product, or process disclosed, or represents that its use would not infringe privately owned rights. Reference herein to any specific commercial product, process, or service by trade name, trademark, manufacturer, or otherwise does not necessarily constitute or imply its endorsement, recommendation, or favoring by the United States Government or any agency thereof, or Battelle Memorial Institute. The views and opinions of authors expressed herein do not necessarily state or reflect those of the United States Government or any agency thereof.

\author{
PACIFIC NORTHWEST NATIONAL LABORATORY \\ operated by \\ BATTELLE \\ for the \\ UNITED STATES DEPARTMENT OF ENERGY \\ under Contract DE-AC05-76RL01830
}

Printed in the United States of America
Available to DOE and DOE contractors from the Office of Scientific and Technical Information,
P.O. Box 62, Oak Ridge, TN 37831-0062;
ph: (865) 576-8401
fax: $(865)$ 576-5728
email: reports@adonis.osti.gov

\begin{abstract}
Available to the public from the National Technical Information Service, U.S. Department of Commerce, 5285 Port Royal Rd., Springfield, VA 22161 ph: (800) 553-6847 fax: $(703) 605-6900$ email: orders@ntis.fedworld.gov online ordering: http://www.ntis.gov/ordering.htm
\end{abstract}

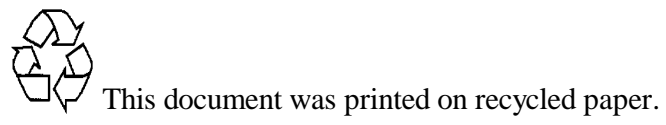




\section{0-DV-10U Sediment and Pore Water Analysis and Report for Samples at Borehole C8096}

M Lindberg

October 2011

Prepared for the U.S. Department of Energy under Contract DE-AC05-76RL01830

Pacific Northwest National Laboratory

Richland, Washington 99352 
10/28/11 11:55

To: Virginia Rohay

From: Michael J. Lindberg

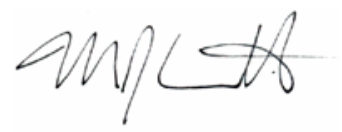

Environmental Sciences Laboratory

Energy and Environment Directorate, Pacific Northwest National Laboratory

Subject: Analytical Data Report for Sediment Samples Collected From 200-DV-1 OU Borehole C8096, Sample Delivery Group ESL090025, SAF Number F11-070

This letter contains the following information for sample delivery group ESL090025

- Cover Sheet

- Narrative

- Analytical Results

- Quality Control

- Geologic Logs

- Geologic Photos

- Chain of Custodies 


\section{Introduction}

On August 30, 2011 sediment samples were received from 200-DV-1 OU Borehole C8096 for geochemical studies.

\section{Analytical Results/Methodology}

The analyses for this project were performed at the 331 building located in the 300 Area of the Hanford Site. The analyses were performed according to Pacific Northwest National Laboratory (PNNL) approved procedures and/or nationally recognized test procedures. The data sets include the sample identification numbers, analytical results, estimated quantification limits (EQL), and quality control data.

\section{Quality Control}

The preparatory and analytical quality control requirements, calibration requirements, acceptance criteria, and failure actions are defined in the on-line QA plan "Conducting Analytical Work in Support of Regulatory Programs" (CAW). This QA plan implements the Hanford Analytical Services Quality Assurance Requirements Documents (HASQARD) for PNNL.

\section{Definitions}

Dup Duplicate

RPD Relative Percent Difference

NR No Recovery (percent recovery less than zero)

ND Non-Detectable

$\%$ REC Percent Recovery

\section{Sample Receipt}

Samples were received with a chain of custody (COC) and were analyzed according to the sample identification numbers supplied by the client. All Samples were refrigerated upon receipt until prepared for analysis.

All samples were received with custody seals intact unless noted in the Case Narrative.

\section{Holding Times}

Holding time is defined as the time from sample preparation to the time of analyses. The prescribed holding times were met for all analytes unless noted in the Case Narrative.

\section{Analytical Results}

All reported analytical results meet the requirements of the CAW or client specified SOW unless noted in the case narrative. 


\section{Case Narrative Report}

\section{Hold Time:}

Due to the requirements of the statement of work and sampling events in the field, the 28 day and the 48 hr requirements could not be met. All applicable hold times were started from the time of preparation and not the time of sampling.

\section{Preparation Blank (PB):}

No discrepancies noted.

\section{Duplicate (DUP):}

No discrepancies noted.

\section{Laboratory Control Samples (LCS):}

No discrepancies noted.

\section{Post Spike (PS):}

Post-Spike Recovery for Calcium (NR) was outside acceptance limits (75-125) in 1J17002-PS1 for ICP-OES Vadose-AE The native concentration of the sample was greater than 5 times the spike concentration. There should be no impact to data as reported.

Post-Spike Recovery for Magnesium (NR) was outside acceptance limits (75-125) in 1J17002-PS1 for ICP-OES Vadose-AE The native concentration of the sample was greater than 5 times the spike concentration. There should be no impact to data as reported.

\section{Matrix Spike (MS):}

No discrepancies noted.

\section{Other QC Criteria:}

No discrepancies noted. 
I certify that this data package is in compliance with the SOW, both technically and for completeness, for other than the conditions detailed above. Release of the data contained in this hard copy data package has been authorized by the Laboratory Analytical Manager as verified by this signature.

\author{
Michael Lindberg
}

\title{
DISCLAIMER
}

This report was prepared as an account of work sponsored by an agency of the United States Government. Neither the United States Government nor any agency thereof, nor Battelle Memorial Institute, nor any of their employees, makes any warranty, express or implied, or assumes any legal liability or responsibility for the accuracy, completeness, or usefulness of any information, apparatus, product, or process disclosed, or represents that its use would not infringe privately owned rights. Reference herein to any specific commercial product, process, or service by trade name, trademark, manufacturer, or otherwise does not necessarily constitute or imply its endorsement, recommendation, or favoring by the United States Government or any agency thereof, or Battelle Memorial Institute. The views and opinions of authors expressed herein do not necessarily state or reflect those of the United States Government or any agency thereof.

\author{
PACIFIC NORTHWEST NATIONAL LABORATORY \\ operated by \\ BATTELLE \\ for the \\ UNITED STATES DEPARTMENT OF ENERGY \\ under Contract DE-AC05-76RLOJ830
}


The following analyses were performed on the following samples included in this report:

Metals 1:1 DI Water Extract by ICPMS

Alkalinity, Titrimetic ( $\mathrm{pH} 4.5)$

Anions By Ion Chromatography

Carbon, Total, Combustion or Oxidation

Inorganic Carbon, Total, Combustion or Oxidation

Iodine-129 1:1 DI Water Extract by ICPMS

Metals 1:1 Water Extract by ICPOES

Metals Acid Extract by ICPOES

Moisture Content

Particle Size Distribution (Dry Sieve)

$\mathrm{pH}$ of Waters By Electrode

Specific Conductance

Tc_U 1:1 DI Water Extract by ICPMS

Total Carbon NP Soil

\section{SAMPLES ANALYZED IN THIS REPORT}

$\begin{array}{lll}\text { HEIS No. } & \text { Laboratory ID } & \text { Matrix } \\ \text { B2CP73 } & 1108012-01 & \text { SOIL } \\ \text { B2CP74 } & 1108012-02 & \text { SOIL }\end{array}$

Date Collected
$\begin{array}{ll}8 / 23 / 11 & 12: 30 \\ 8 / 23 / 11 & 13: 25\end{array}$

Date Received

8/30/11 14:40

$8 / 30 / 11 \quad 14: 40$ 


\section{Wet Chemistry}

Alkalinity as CaCO3 (ug/g dry) by Standard Methods 2320B

Lab ID HEIS No.

Results

Analyzed

Batch

1108012-01

$\mathrm{B} 2 \mathrm{CP} 73$

$8.22 \mathrm{E} 1$

$3.25 \mathrm{E} 1$

$10 / 13 / 11$

$1 \mathrm{~J} 12003$

$1108012-02$

$\mathrm{B} 2 \mathrm{CP} 74$

9.05E1 2.77E1

10/13/11

1J12003 


\section{Wet Chemistry}

Specific Conductance (EC) (mS/cm) by EPA 120.1

Lab ID HEIS No.

Results

Analyzed

Batch

1108012-01

$\mathrm{B} 2 \mathrm{CP} 73$

2.39E-1

$10 / 12 / 11$

$1 \mathrm{~J} 12002$

1108012-02

$\mathrm{B} 2 \mathrm{CP} 74$

$2.13 \mathrm{E}-1 \quad 1.00 \mathrm{E}-2$

$10 / 12 / 11$

$1 \mathrm{~J} 12002$ 


\section{Wet Chemistry}

Moisture Content (\% by Weight) by AGG-WC-001

Lab ID HEIS No.

Results

EQL

Analyzed

Batch

1108012-01

$\mathrm{B} 2 \mathrm{CP} 73$

$1.91 \mathrm{E} 1$

$\mathrm{N} / \mathrm{A}$

$10 / 07 / 11$

1J07003

$1108012-02$

$\mathrm{B} 2 \mathrm{CP} 74$

$8.93 \mathrm{E} 0$

N/A

10/07/11

1J07003 


\section{Wet Chemistry}

pH (pH Units) by AGG-pH-001

Lab ID HEIS No.

Results

EQL

Analyzed

Batch

1108012-01

B2CP73

7.04E0

$\mathrm{N} / \mathrm{A}$

$10 / 12 / 11$

$1 \mathrm{~J} 12001$

1108012-02

$\mathrm{B} 2 \mathrm{CP} 74$

$7.41 \mathrm{E} 0$

N/A

$10 / 12 / 11$

1J12001 


\section{Anions by Ion Chromatography}

\begin{tabular}{|lccccccl|}
\hline CAS \# & Analyte & Results & Units & EQL & Analyzed & Batch & Method \\
\hline HEIS No. & B2CP73 & \multicolumn{2}{c|}{ Lab ID: } & $\mathbf{1 1 0 8 0 1 2 - 0 1}$ & & & \\
$16984-48-8$ & Fluoride & $<1.00 \mathrm{E} 0$ & ug/g dry & $1.00 \mathrm{E} 0$ & $10 / 12 / 11$ & $1 \mathrm{~J} 12004$ & AGG-IC-001 \\
$16887-00-6$ & Chloride & $9.45 \mathrm{E} 0$ & ug/g dry & $2.50 \mathrm{E} 0$ & $10 / 12 / 11$ & $1 \mathrm{~J} 12004$ & AGG-IC-001 \\
$14797-55-8$ & Nitrate & $3.42 \mathrm{E} 1$ & ug/g dry & $5.00 \mathrm{E} 0$ & $10 / 12 / 11$ & $1 \mathrm{~J} 12004$ & AGG-IC-001 \\
$14808-79-8$ & Sulfate & $2.25 \mathrm{E} 1$ & ug/g dry & $7.51 \mathrm{E} 0$ & $10 / 12 / 11$ & $1 \mathrm{~J} 12004$ & AGG-IC-001 \\
$14265-44-2$ & Phosphate & $<7.51 \mathrm{E} 0$ & ug/g dry & $7.51 \mathrm{E} 0$ & $10 / 12 / 11$ & $1 \mathrm{~J} 12004$ & AGG-IC-001 \\
\hline HEIS No. & B2CP74 & \multicolumn{2}{c}{ Lab ID: $\mathbf{1 1 0 8 0 1 2 - 0 2}$} & & & \\
$16984-48-8$ & Fluoride & $<1.00 \mathrm{E} 0$ & ug/g dry & $1.00 \mathrm{E} 0$ & $10 / 12 / 11$ & $1 \mathrm{~J} 12004$ & AGG-IC-001 \\
$16887-00-6$ & Chloride & $3.66 \mathrm{E} 0$ & ug/g dry & $2.50 \mathrm{E} 0$ & $10 / 12 / 11$ & $1 \mathrm{~J} 12004$ & AGG-IC-001 \\
$14797-55-8$ & Nitrate & $1.56 \mathrm{E} 1$ & ug/g dry & $5.00 \mathrm{E} 0$ & $10 / 12 / 11$ & $1 \mathrm{~J} 12004$ & AGG-IC-001 \\
$14808-79-8$ & Sulfate & $1.89 \mathrm{E} 1$ & ug/g dry & $7.50 \mathrm{E} 0$ & $10 / 12 / 11$ & $1 \mathrm{~J} 12004$ & AGG-IC-001 \\
$14265-44-2$ & Phosphate & $<7.50 \mathrm{E} 0$ & ug/g dry & $7.50 \mathrm{E} 0$ & $10 / 12 / 11$ & $1 \mathrm{~J} 12004$ & AGG-IC-001 \\
\hline
\end{tabular}


Total Metals by PNNL-AGG-ICP-AES/1:1 Water Extract

\begin{tabular}{|c|c|c|c|c|c|c|c|}
\hline CAS \# & Analyte & Results & Units & EQL & Analyzed & Batch & Method \\
\hline HEIS No. & В2СР73 & \multicolumn{3}{|c|}{ Lab ID: $\quad$ 1108012-01 } & & & \\
\hline $7440-39-3$ & Barium & $<1.24 \mathrm{E}-1$ & ug/g dry & $1.24 \mathrm{E}-1$ & $10 / 17 / 11$ & $1 \mathrm{~J} 17001$ & PNNL-AGG-ICP-AES \\
\hline HEIS No. & В2CР74 & \multicolumn{3}{|c|}{ Lab ID: $\quad$ 1108012-02 } & & & \\
\hline $7440-39-3$ & Barium & $<1.24 \mathrm{E}-1$ & ug/g dry & $1.24 \mathrm{E}-1$ & $10 / 17 / 11$ & $1 \mathrm{~J} 17001$ & PNNL-AGG-ICP-AES \\
\hline
\end{tabular}


Total Metals by PNNL-AGG-ICP-AES/Acid Extract

\begin{tabular}{|lccccccl|}
\hline CAS \# & Analyte & Results & Units & EQL & Analyzed & Batch & Method \\
\hline HEIS No. & B2CP73 & \multicolumn{2}{c}{ Lab ID: } & $\mathbf{1 1 0 8 0 1 2 - 0 1}$ & & & \\
$7440-70-2$ & Calcium & $7.54 \mathrm{E} 3$ & ug/g dry & $2.88 \mathrm{E} 1$ & $10 / 17 / 11$ & $1 \mathrm{~J} 17002$ & PNNL-AGG-ICP-AES \\
$7440-09-7$ & Potassium & $1.35 \mathrm{E} 3$ & ug/g dry & $1.06 \mathrm{E} 2$ & $10 / 17 / 11$ & $1 \mathrm{~J} 17002$ & PNNL-AGG-ICP-AES \\
$7439-95-4$ & Magnesium & $4.33 \mathrm{E} 3$ & ug/g dry & $7.90 \mathrm{E} 0$ & $10 / 17 / 11$ & $1 \mathrm{~J} 17002$ & PNNL-AGG-ICP-AES \\
$7440-23-5$ & Sodium & $1.70 \mathrm{E} 2$ & ug/g dry & $7.81 \mathrm{E} 1$ & $10 / 17 / 11$ & $1 \mathrm{~J} 17002$ & PNNL-AGG-ICP-AES \\
\hline HEIS No. & B2CP74 & \multicolumn{2}{c}{ Lab ID: $\mathbf{1 1 0 8 0 1 2 - 0 2}$} & & & \\
$7440-70-2$ & Calcium & $4.26 \mathrm{E} 4$ & ug/g dry & $2.61 \mathrm{E} 1$ & $10 / 17 / 11$ & $1 \mathrm{~J} 17002$ & PNNL-AGG-ICP-AES \\
$7440-09-7$ & Potassium & $7.63 \mathrm{E} 2$ & ug/g dry & $9.64 \mathrm{E} 1$ & $10 / 17 / 11$ & $1 \mathrm{~J} 17002$ & PNNL-AGG-ICP-AES \\
$7439-95-4$ & Magnesium & $3.97 \mathrm{E} 3$ & ug/g dry & $7.17 \mathrm{E} 0$ & $10 / 17 / 11$ & $1 \mathrm{~J} 17002$ & PNNL-AGG-ICP-AES \\
$7440-23-5$ & Sodium & $3.25 \mathrm{E} 2$ & ug/g dry & $7.09 \mathrm{E} 1$ & $10 / 17 / 11$ & $1 \mathrm{~J} 17002$ & PNNL-AGG-ICP-AES \\
\hline
\end{tabular}


Radionuclides by ICP-MS/1:1 Water Extract

\begin{tabular}{|c|c|c|c|c|c|c|c|}
\hline CAS \# & Analyte & Results & Units & EQL & Analyzed & Batch & Method \\
\hline HEIS No. & B2CP73 & \multicolumn{3}{|c|}{ Lab ID: $\quad$ 1108012-01 } & & & \\
\hline $15046-84-1$ & Iodine-129 & $<7.44 \mathrm{E}-3$ & ug/g dry & $7.44 \mathrm{E}-3$ & $10 / 25 / 11$ & $1 \mathrm{~J} 21002$ & PNNL-AGG-415 \\
\hline HEIS No. & B2CP74 & \multicolumn{3}{|c|}{ Lab ID: $\quad 1108012-02$} & & & \\
\hline $15046-84-1$ & Iodine-129 & $<7.44 \mathrm{E}-3$ & ug/g dry & $7.44 \mathrm{E}-3$ & $10 / 25 / 11$ & $1 \mathrm{~J} 21002$ & PNNL-AGG-415 \\
\hline
\end{tabular}


Radionuclides by ICP-MS/1:1 Water Extract

\begin{tabular}{|c|c|c|c|c|c|c|c|}
\hline CAS \# & Analyte & Results & Units & EQL & Analyzed & Batch & Method \\
\hline HEIS No. & В2CP73 & \multicolumn{3}{|c|}{ Lab ID: $\quad 1108012-01$} & & & \\
\hline $14133-76-7$ & Technetium-99 & $<7.81 \mathrm{E}-5$ & ug/g dry & $7.81 \mathrm{E}-5$ & $10 / 20 / 11$ & $1 \mathrm{~J} 20001$ & PNNL-AGG-415 \\
\hline $\mathrm{U}-238$ & Uranium 238 & $1.04 \mathrm{E}-3$ & ug/g dry & $1.60 \mathrm{E}-4$ & $10 / 20 / 11$ & $1 \mathrm{~J} 20001$ & PNNL-AGG-415 \\
\hline HEIS No. & B2CP74 & \multicolumn{3}{|c|}{ Lab ID: $\quad$ 1108012-02 } & & & \\
\hline $14133-76-7$ & Technetium-99 & $<7.80 \mathrm{E}-5$ & ug/g dry & $7.80 \mathrm{E}-5$ & $10 / 20 / 11$ & $1 \mathrm{~J} 20001$ & PNNL-AGG-415 \\
\hline U-238 & Uranium 238 & $9.40 \mathrm{E}-3$ & ug/g dry & $1.60 \mathrm{E}-4$ & $10 / 20 / 11$ & $1 \mathrm{~J} 20001$ & PNNL-AGG-415 \\
\hline
\end{tabular}


RCRA Metals By PNNL-AGG-415/1:1 Water Extract

\begin{tabular}{|c|c|c|c|c|c|c|c|}
\hline CAS \# & Analyte & Results & Units & EQL & Analyzed & Batch & Method \\
\hline HEIS No. & В2CР73 & \multicolumn{3}{|c|}{ Lab ID: $\quad$ 1108012-01 } & & & \\
\hline $14092-98-9$ & Chromium & $<6.89 \mathrm{E}-3$ & ug/g dry & $6.89 \mathrm{E}-3$ & $10 / 13 / 11$ & $1 J 13002$ & PNNL-AGG-415 \\
\hline $7440-38-2$ & Arsenic & $5.91 \mathrm{E}-3$ & ug/g dry & $5.68 \mathrm{E}-3$ & 10/13/11 & $1 \mathrm{~J} 13002$ & PNNL-AGG-415 \\
\hline $14687-58-2$ & Selenium & $<1.58 \mathrm{E}-2$ & ug/g dry & $1.58 \mathrm{E}-2$ & $10 / 13 / 11$ & $1 \mathrm{~J} 13002$ & PNNL-AGG-415 \\
\hline $14378-37-1$ & Silver & $<6.26 \mathrm{E}-3$ & ug/g dry & $6.26 \mathrm{E}-3$ & 10/13/11 & $1 \mathrm{~J} 13002$ & PNNL-AGG-415 \\
\hline $14041-58-8$ & Cadmium & $<7.72 \mathrm{E}-4$ & $\mathrm{ug} / \mathrm{g}$ dry & $7.72 \mathrm{E}-4$ & $10 / 13 / 11$ & $1 J 13002$ & PNNL-AGG-415 \\
\hline $13966-27-3$ & Lead & $<2.71 \mathrm{E}-3$ & $\mathrm{ug} / \mathrm{g}$ dry & $2.71 \mathrm{E}-3$ & $10 / 13 / 11$ & $1 J 13002$ & PNNL-AGG-415 \\
\hline $14191-86-7$ & Mercury & $<1.46 \mathrm{E}-3$ & ug/g dry & $1.46 \mathrm{E}-3$ & $10 / 13 / 11$ & $1 J 13002$ & PNNL-AGG-415 \\
\hline HEIS No. & B2CР74 & \multicolumn{3}{|c|}{ Lab ID: $\quad 1108012-02$} & & & \\
\hline $14092-98-9$ & Chromium & $<6.89 \mathrm{E}-3$ & ug/g dry & $6.89 \mathrm{E}-3$ & $10 / 13 / 11$ & $1 J 13002$ & PNNL-AGG-415 \\
\hline $7440-38-2$ & Arsenic & $9.46 \mathrm{E}-3$ & ug/g dry & $5.67 \mathrm{E}-3$ & 10/13/11 & $1 \mathrm{~J} 13002$ & PNNL-AGG-415 \\
\hline $14687-58-2$ & Selenium & $<1.58 \mathrm{E}-2$ & ug/g dry & $1.58 \mathrm{E}-2$ & $10 / 13 / 11$ & $1 \mathrm{~J} 13002$ & PNNL-AGG-415 \\
\hline $14378-37-1$ & Silver & $<6.26 \mathrm{E}-3$ & ug/g dry & $6.26 \mathrm{E}-3$ & $10 / 13 / 11$ & $1 \mathrm{~J} 13002$ & PNNL-AGG-415 \\
\hline $14041-58-8$ & Cadmium & $<7.71 \mathrm{E}-4$ & ug/g dry & $7.71 \mathrm{E}-4$ & $10 / 13 / 11$ & $1 J 13002$ & PNNL-AGG-415 \\
\hline $13966-27-3$ & Lead & $<2.71 \mathrm{E}-3$ & $\mathrm{ug} / \mathrm{g}$ dry & $2.71 \mathrm{E}-3$ & $10 / 13 / 11$ & $1 J 13002$ & PNNL-AGG-415 \\
\hline $14191-86-7$ & Mercury & $<1.46 \mathrm{E}-3$ & ug/g dry & $1.46 \mathrm{E}-3$ & $10 / 13 / 11$ & $1 \mathrm{~J} 13002$ & PNNL-AGG-415 \\
\hline
\end{tabular}




\section{Carbon Analysis/Soil}

Total Carbon (ug/g) by AGG-TOC-001

Lab ID HEIS No.

Results

EQL

Analyzed

Batch

1108012-01

$\mathrm{B} 2 \mathrm{CP} 73$

$2.73 \mathrm{E} 3$

$2.00 \mathrm{E} 2$

10/10/11

$1 \mathrm{~J} 10002$

$1108012-02$

$\mathrm{B} 2 \mathrm{CP} 74$

$1.61 \mathrm{E} 4 \quad 2.00 \mathrm{E} 2$

$10 / 10 / 11$

1J10002 


\section{Carbon Analysis/Soil}

Total Inorganic Carbon (ug/g) by AGG-TOC-001

Lab ID

HEIS No.

Results

Analyzed

Batch

1108012-01

$\mathrm{B} 2 \mathrm{CP} 73$

2.81E3

$2.94 \mathrm{E} 2$

$10 / 10 / 11$

$1 \mathrm{~J} 10003$

$1108012-02$

$\mathrm{B} 2 \mathrm{CP} 74$

$1.52 \mathrm{E} 4 \quad 2.94 \mathrm{E} 2$

$10 / 10 / 11$

$1 \mathrm{~J} 10003$ 


\section{Carbon Analysis/Soil}

Total Organic Carbon (ug/g) by ASTM E1915

Lab ID HEIS No.

\begin{tabular}{cccc} 
Results & EQL & Analyzed & Batch \\
\hline$<4.94 \mathrm{E} 2$ & $4.94 \mathrm{E} 2$ & $10 / 10 / 11$ & {$[$ CALC] } \\
$8.88 \mathrm{E} 2$ & $4.94 \mathrm{E} 2$ & $10 / 10 / 11$ & {$[$ CALC] }
\end{tabular}

1108012-01

$\mathrm{B} 2 \mathrm{CP} 73$

$8.88 \mathrm{E} 2$

$4.94 \mathrm{E} 2$

$10 / 10 / 11$

$[\mathrm{CALC}]$ 


\section{Particle Size Distribution (Dry Sieve)}

\begin{tabular}{|c|c|c|c|c|c|c|c|}
\hline CAS \# & Analyte & Results & Units & EQL & Analyzed & Batch & Method \\
\hline HEIS No. & B2CP73 & \multicolumn{3}{|c|}{ Lab ID: $\quad$ 1108012-01 } & & & \\
\hline PAS2.5IN & Particle Size 63000 uM & $1.00 \mathrm{E} 2$ & $\%$ Passing & N/A & $10 / 15 / 11$ & $1 \mathrm{~J} 10004$ & ASTM D422-63 \\
\hline PAS1.25IN & Particle Size $31500 \mathrm{uM}$ & $1.00 \mathrm{E} 2$ & $\%$ Passing & N/A & $10 / 15 / 11$ & $1 \mathrm{~J} 10004$ & ASTM D422-63 \\
\hline PAS5/8IN & Particle Size $16000 \mathrm{uM}$ & $1.00 \mathrm{E} 2$ & $\%$ Passing & N/A & $10 / 15 / 11$ & $1 \mathrm{~J} 10004$ & ASTM D422-63 \\
\hline $\mathrm{PAS} 5 / 16 \mathrm{IN}$ & Particle Size 8000 uM & $1.00 \mathrm{E} 2$ & $\%$ Passing & N/A & $10 / 15 / 11$ & $1 \mathrm{~J} 10004$ & ASTM D422-63 \\
\hline PAS\#5 & Particle Size $4000 \mathrm{uM}$ & $1.00 \mathrm{E} 2$ & $\%$ Passing & N/A & $10 / 15 / 11$ & $1 \mathrm{~J} 10004$ & ASTM D422-63 \\
\hline PAS\#10 & Particle Size 2000 uM & $9.99 \mathrm{E} 1$ & $\%$ Passing & N/A & $10 / 15 / 11$ & $1 \mathrm{~J} 10004$ & ASTM D422-63 \\
\hline PAS\#18 & Particle Size $1000 \mathrm{uM}$ & $9.92 \mathrm{E} 1$ & $\%$ Passing & N/A & $10 / 15 / 11$ & $1 \mathrm{~J} 10004$ & ASTM D422-63 \\
\hline PAS\#35 & Particle Size 500 uM & $9.87 \mathrm{E} 1$ & $\%$ Passing & N/A & $10 / 15 / 11$ & $1 \mathrm{~J} 10004$ & ASTM D422-63 \\
\hline PAS $\# 60$ & Particle Size $250 \mathrm{uM}$ & $9.76 \mathrm{E} 1$ & $\%$ Passing & N/A & $10 / 15 / 11$ & $1 \mathrm{~J} 10004$ & ASTM D422-63 \\
\hline PAS\#120 & Particle Size $125 \mathrm{uM}$ & $9.27 \mathrm{E} 1$ & $\%$ Passing & N/A & $10 / 15 / 11$ & $1 J 10004$ & ASTM D422-63 \\
\hline PAS\#230 & Particle Size 63 uM & $5.73 \mathrm{E} 1$ & $\%$ Passing & N/A & $10 / 15 / 11$ & $1 \mathrm{~J} 10004$ & ASTM D422-63 \\
\hline HEIS No. & B2CP74 & \multicolumn{3}{|c|}{ Lab ID: $\quad$ 1108012-02 } & & & \\
\hline PAS2.5IN & Particle Size 63000 uM & $1.00 \mathrm{E} 2$ & $\%$ Passing & N/A & $10 / 15 / 11$ & $1 J 10004$ & ASTM D422-63 \\
\hline PAS1.25IN & Particle Size $31500 \mathrm{uM}$ & $8.36 \mathrm{E} 1$ & $\%$ Passing & N/A & $10 / 15 / 11$ & $1 J 10004$ & ASTM D422-63 \\
\hline PAS5/8IN & Particle Size $16000 \mathrm{uM}$ & 4.93E1 & $\%$ Passing & N/A & $10 / 15 / 11$ & $1 \mathrm{~J} 10004$ & ASTM D422-63 \\
\hline PAS5/16IN & Particle Size 8000 uM & $3.89 \mathrm{E} 1$ & $\%$ Passing & N/A & $10 / 15 / 11$ & $1 J 10004$ & ASTM D422-63 \\
\hline PAS\#5 & Particle Size $4000 \mathrm{uM}$ & $3.41 \mathrm{E} 1$ & $\%$ Passing & N/A & $10 / 15 / 11$ & $1 \mathrm{~J} 10004$ & ASTM D422-63 \\
\hline PAS\#10 & Particle Size $2000 \mathrm{uM}$ & $2.99 \mathrm{E} 1$ & $\%$ Passing & N/A & $10 / 15 / 11$ & $1 \mathrm{~J} 10004$ & ASTM D422-63 \\
\hline PAS\#18 & Particle Size $1000 \mathrm{uM}$ & $1.95 \mathrm{E} 1$ & $\%$ Passing & N/A & $10 / 15 / 11$ & $1 \mathrm{~J} 10004$ & ASTM D422-63 \\
\hline PAS\#35 & Particle Size 500 uM & $1.13 \mathrm{E} 1$ & $\%$ Passing & N/A & $10 / 15 / 11$ & $1 \mathrm{~J} 10004$ & ASTM D422-63 \\
\hline PAS\#60 & Particle Size $250 \mathrm{uM}$ & $6.50 \mathrm{E} 0$ & $\%$ Passing & N/A & $10 / 15 / 11$ & $1 \mathrm{~J} 10004$ & ASTM D422-63 \\
\hline PAS\#120 & Particle Size $125 \mathrm{uM}$ & $3.37 \mathrm{E} 0$ & $\%$ Passing & N/A & $10 / 15 / 11$ & $1 \mathrm{~J} 10004$ & ASTM D422-63 \\
\hline PAS\#230 & Particle Size 63 uM & $1.54 \mathrm{E} 0$ & $\%$ Passing & N/A & $10 / 15 / 11$ & $1 J 10004$ & ASTM D422-63 \\
\hline
\end{tabular}




\section{PARTICLE SIZE DISTRIBUTION ANALYSIS HYDROMETER}

$1108012-01$ (B2CP73)
\begin{tabular}{|c|c|}
\hline $\begin{array}{c}\text { Particle } \\
\text { Size (uM) }\end{array}$ & $\begin{array}{c}\% \\
\text { Passing }\end{array}$ \\
\hline 96.4 & 92.9 \\
\hline 66.6 & 79.4 \\
\hline 36.6 & 52.3 \\
\hline 19.0 & 27.1 \\
\hline 10.7 & 17.42 \\
\hline 7.54 & 14.52 \\
\hline 6.12 & 11.61 \\
\hline 5.29 & 10.65 \\
\hline 1.52 & 6.78 \\
\hline
\end{tabular}

$1108012-02$ (B2CP74)
\begin{tabular}{|c|c|}
\hline $\begin{array}{c}\text { Particle } \\
\text { Size (uM) }\end{array}$ & $\%$ Passing \\
\hline 91.0 & 23.6 \\
\hline 63.6 & 21.5 \\
\hline 35.9 & 17.8 \\
\hline 19.1 & 13.3 \\
\hline 10.8 & 9.97 \\
\hline 7.54 & 8.16 \\
\hline 6.12 & 7.25 \\
\hline 5.25 & 6.04 \\
\hline 1.49 & 3.63 \\
\hline
\end{tabular}


Wet Chemistry - Quality Control

Environmental Science Laboratory

\begin{tabular}{|lrrrrrrrrrrr}
\hline & & Reporting & & Spike & Source & & \%REC & & RPD \\
Analyte & Result & Limit & Units & Level & Result & $\%$ REC & Limits & RPD & Limit & Notes \\
\hline
\end{tabular}

Batch 1J12001 - 1:1 Water Extract (pH_EC_Alk)

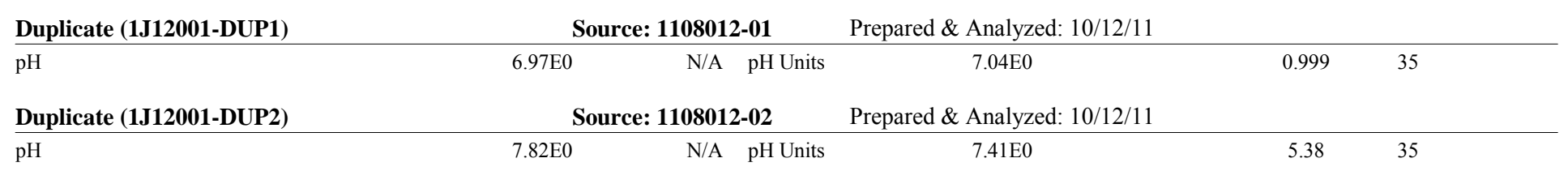

Batch 1J12002 - 1:1 Water Extract (pH_EC_Alk)

\begin{tabular}{|c|c|c|c|c|c|c|c|}
\hline \multirow{2}{*}{$\frac{\text { Blank (1J12002-BLK1) }}{\text { Specific Conductance (EC) }}$} & & & & \multicolumn{4}{|c|}{ Prepared \& Analyzed: 10/12/11 } \\
\hline & $<1.00 \mathrm{E}-2$ & $1.00 \mathrm{E}-2$ & $\mathrm{mS} / \mathrm{cm}$ & & & & \\
\hline Duplicate (1J12002-DUP1) & \multicolumn{3}{|c|}{ Source: 1108012-01 } & \multicolumn{4}{|c|}{ Prepared \& Analyzed: 10/12/11 } \\
\hline Specific Conductance (EC) & $2.43 \mathrm{E}-1$ & $1.00 \mathrm{E}-2$ & $\mathrm{mS} / \mathrm{cm}$ & $2.39 \mathrm{E}-1$ & & 1.41 & 35 \\
\hline Duplicate (1J12002-DUP2) & \multicolumn{3}{|c|}{ Source: 1108012-02 } & \multicolumn{4}{|c|}{ Prepared \& Analyzed: 10/12/11 } \\
\hline Specific Conductance (EC) & $2.25 \mathrm{E}-1$ & $1.00 \mathrm{E}-2$ & $\mathrm{mS} / \mathrm{cm}$ & $2.13 \mathrm{E}-1$ & & 5.30 & 35 \\
\hline \multicolumn{8}{|c|}{ Batch 1J12003 - 1:1 Water Extract (pH_EC_Alk) } \\
\hline Blank (1J12003-BLK1) & & & & Prepared: 10/12/11 & Analyzed: 10/13/11 & & \\
\hline Alkalinity as $\mathrm{CaCO} 3$ & $<2.35 \mathrm{E} 1$ & $2.35 \mathrm{E} 1$ & ug/g wet & & & & \\
\hline Duplicate (1J12003-DUP1) & \multicolumn{3}{|c|}{ Source: 1108012-01 } & Prepared: 10/12/11 & Analyzed: 10/13/11 & & \\
\hline Alkalinity as $\mathrm{CaCO} 3$ & $9.17 \mathrm{E} 1$ & $3.25 \mathrm{E} 1$ & ug/g dry & $8.22 \mathrm{E} 1$ & & 10.9 & 35 \\
\hline Duplicate (1J12003-DUP2) & \multicolumn{3}{|c|}{ Source: 1108012-02 } & Prepared: 10/12/11 & Analyzed: 10/13/11 & & \\
\hline Alkalinity as $\mathrm{CaCO} 3$ & $9.35 \mathrm{E} 1$ & $2.77 \mathrm{E} 1$ & ug/g dry & $9.05 \mathrm{E} 1$ & & 3.33 & 35 \\
\hline
\end{tabular}


Anions by Ion Chromatography - Quality Control

Environmental Science Laboratory

\begin{tabular}{|lrrrrrrrrrrr}
\hline & & Reporting & & Spike & Source & & & $\%$ REC & & RPD & \\
Analyte & Result & Limit & Units & Level & Result & $\%$ REC & Limits & RPD & Limit & Notes \\
\hline
\end{tabular}

Batch 1J12004 - 1:1 Water Extract (IC)

\begin{tabular}{|c|c|c|c|c|c|c|c|c|c|}
\hline \multirow{2}{*}{$\begin{array}{l}\text { Blank (1J12004-BLK1) } \\
\text { Fluoride }\end{array}$} & \multicolumn{9}{|c|}{ Prepared \& Analyzed: 10/12/11 } \\
\hline & $<1.00 \mathrm{E}-1$ & $1.00 \mathrm{E}-1$ & ug/g wet & & & & & & \\
\hline Chloride & $<2.50 \mathrm{E}-1$ & $2.50 \mathrm{E}-1$ & $"$ & & & & & & \\
\hline Nitrate & $<5.00 \mathrm{E}-1$ & $5.00 \mathrm{E}-1$ & $"$ & & & & & & \\
\hline Sulfate & $<7.50 \mathrm{E}-1$ & $7.50 \mathrm{E}-1$ & $"$ & & & & & & \\
\hline Phosphate & $<7.50 \mathrm{E}-1$ & $7.50 \mathrm{E}-1$ & $"$ & & & & & & \\
\hline LCS (1J12004-BS1) & \multicolumn{9}{|c|}{ Prepared \& Analyzed: 10/12/11 } \\
\hline Fluoride & $2.01 \mathrm{E} 0$ & $1.00 \mathrm{E}-1$ & ug/g wet & $2.00 \mathrm{E} 0$ & & 101 & $80-120$ & & \\
\hline Chloride & $4.99 \mathrm{E} 0$ & $2.50 \mathrm{E}-1$ & $"$ & $5.01 \mathrm{E} 0$ & & 99.7 & $80-120$ & & \\
\hline Nitrate & $1.07 \mathrm{E} 1$ & $5.00 \mathrm{E}-1$ & $"$ & $1.00 \mathrm{E} 1$ & & 107 & $80-120$ & & \\
\hline Sulfate & $1.51 \mathrm{E} 1$ & $7.50 \mathrm{E}-1$ & $"$ & $1.50 \mathrm{E} 1$ & & 101 & $80-120$ & & \\
\hline Phosphate & $1.49 \mathrm{E} 1$ & $7.50 \mathrm{E}-1$ & $"$ & $1.50 \mathrm{E} 1$ & & 98.9 & $80-120$ & & \\
\hline Duplicate (1J12004-DUP1) & \multicolumn{3}{|c|}{ Source: $1108012-01$} & \multicolumn{6}{|c|}{ Prepared \& Analyzed: 10/12/11 } \\
\hline Fluoride & $<1.00 \mathrm{E} 0$ & $1.00 \mathrm{E} 0$ & ug/g dry & & ND & & & & 20 \\
\hline Chloride & $9.64 \mathrm{E} 0$ & $2.50 \mathrm{E} 0$ & $"$ & & $9.45 \mathrm{E} 0$ & & & 2.03 & 20 \\
\hline Nitrate & $3.48 \mathrm{E} 1$ & $5.00 \mathrm{E} 0$ & $"$ & & $3.42 \mathrm{E} 1$ & & & 1.97 & 20 \\
\hline Sulfate & $2.25 \mathrm{E} 1$ & $7.50 \mathrm{E} 0$ & $"$ & & $2.25 \mathrm{E} 1$ & & & 0.0273 & 20 \\
\hline Phosphate & $<7.50 \mathrm{E} 0$ & $7.50 \mathrm{E} 0$ & $"$ & & ND & & & & 20 \\
\hline Duplicate (1J12004-DUP2) & \multicolumn{3}{|c|}{ Source: 1108012-02 } & \multicolumn{6}{|c|}{ Prepared \& Analyzed: 10/12/11 } \\
\hline Fluoride & $<9.99 \mathrm{E}-1$ & $9.99 \mathrm{E}-1$ & ug/g dry & & ND & & & & 20 \\
\hline Chloride & $3.70 \mathrm{E} 0$ & $2.50 \mathrm{E} 0$ & $"$ & & $3.66 \mathrm{E} 0$ & & & 0.982 & 20 \\
\hline Nitrate & $1.56 \mathrm{E} 1$ & 4.99E0 & $"$ & & $1.56 \mathrm{E} 1$ & & & 0.536 & 20 \\
\hline Sulfate & $2.04 \mathrm{E} 1$ & 7.49E0 & $"$ & & $1.89 \mathrm{E} 1$ & & & 7.52 & 20 \\
\hline Phosphate & $<7.49 \mathrm{E} 0$ & $7.49 \mathrm{E} 0$ & $"$ & & ND & & & & 20 \\
\hline Post Spike (1J12004-PS1) & \multicolumn{3}{|c|}{ Source: 1108012-01 } & \multicolumn{4}{|c|}{ Prepared \& Analyzed: 10/12/11 } & & \\
\hline Fluoride & $7.82 \mathrm{E}-1$ & N/A & $\mathrm{ug} / \mathrm{mL}$ & $7.69 \mathrm{E}-1$ & $5.50 \mathrm{E}-2$ & 94.5 & $75-125$ & & \\
\hline Chloride & $2.82 \mathrm{E} 0$ & N/A & $"$ & $1.92 \mathrm{E} 0$ & $9.44 \mathrm{E}-1$ & 97.5 & $75-125$ & & \\
\hline Nitrate & 7.22E0 & $\mathrm{N} / \mathrm{A}$ & $"$ & $3.85 \mathrm{E} 0$ & $3.41 \mathrm{E} 0$ & 98.9 & $75-125$ & & \\
\hline Sulfate & $7.98 \mathrm{E} 0$ & N/A & $"$ & $5.77 \mathrm{E} 0$ & $2.25 \mathrm{E} 0$ & 99.2 & $75-125$ & & \\
\hline Phosphate & $5.56 \mathrm{E} 0$ & N/A & $"$ & $5.77 \mathrm{E} 0$ & $2.37 \mathrm{E}-1$ & 92.2 & $75-125$ & & \\
\hline
\end{tabular}


Total Metals by PNNL-AGG-ICP-AES/1:1 Water Extract - Quality Control

Environmental Science Laboratory

\begin{tabular}{|lrrrrrrrrrrr}
\hline & & Reporting & & Spike & Source & & & $\%$ REC & & RPD & \\
Analyte & Result & Limit & Units & Level & Result & $\%$ REC & Limits & RPD & Limit & Notes \\
\hline
\end{tabular}

Batch 1J17001 - 1:1 Water Extract (ICP/ICPMS)

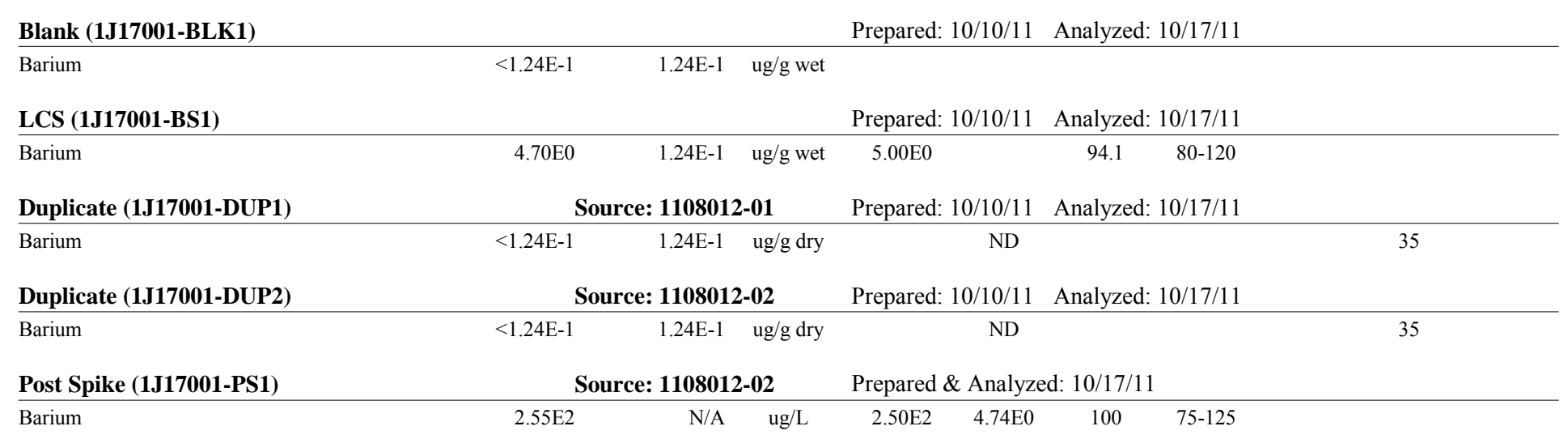


Total Metals by PNNL-AGG-ICP-AES/Acid Extract - Quality Control

Environmental Science Laboratory

\begin{tabular}{|lrrrrrrrrrrr}
\hline & & Reporting & & Spike & Source & & & $\%$ REC & & RPD & \\
Analyte & Result & Limit & Units & Level & Result & $\%$ REC & Limits & RPD & Limit & Notes \\
\hline
\end{tabular}

Batch 1J17002 - ASTM D 5198 (ICP/ICPMS)

Blank (1J17002-BLK1)

Calcium

Potassium

Magnesium

Sodium

LCS (1J17002-BS1)

\section{Calcium}

Potassium

Magnesium

Sodium

Duplicate (1J17002-DUP1)

Calcium

Potassium

Magnesium

Sodium

Duplicate (1J17002-DUP2)

Calcium

Potassium

Magnesium

Sodium

Post Spike (1J17002-PS1)

Calcium

Potassium

Magnesium

Sodium

$\begin{array}{lll}<7.02 \mathrm{E} 0 & 7.02 \mathrm{E} 0 & \text { ug/g wet } \\ <2.59 \mathrm{E} 1 & 2.59 \mathrm{E} 1 & " \\ <1.92 \mathrm{E} 0 & 1.92 \mathrm{E} 0 & " \\ <1.90 \mathrm{E} 1 & 1.90 \mathrm{E} 1 & \end{array}$

Prepared: 10/07/11 Analyzed: 10/17/11

Prepared: 10/07/11 Analyzed: 10/17/11

$\begin{array}{lrcrrr}6.03 \mathrm{E} 0 & 7.02 \mathrm{E}-1 & \text { ug/g wet } & 5.82 \mathrm{E} 0 & 104 & 80-120 \\ 6.16 \mathrm{E} 1 & 2.59 \mathrm{E} 0 & " & 5.82 \mathrm{E} 1 & 106 & 80-120 \\ 5.53 \mathrm{E} 0 & 1.92 \mathrm{E}-1 & " & 5.82 \mathrm{E} 0 & 95.1 & 80-120 \\ 6.51 \mathrm{E} 0 & 1.90 \mathrm{E} 0 & " & 5.82 \mathrm{E} 0 & 112 & 80-120\end{array}$

Source: 1108012-01 Prepared: 10/07/11 Analyzed: 10/17/11

$\begin{array}{lccccc}7.16 \mathrm{E} 3 & 2.89 \mathrm{E} 1 & \text { ug/g dry } & 7.54 \mathrm{E} 3 & 5.19 & 35 \\ 1.36 \mathrm{E} 3 & 1.07 \mathrm{E} 2 & " & 1.35 \mathrm{E} 3 & 0.304 & 35 \\ 4.18 \mathrm{E} 3 & 7.93 \mathrm{E} 0 & " & 4.33 \mathrm{E} 3 & 3.59 & 35 \\ 1.67 \mathrm{E} 2 & 7.85 \mathrm{E} 1 & " & 1.70 \mathrm{E} 2 & 1.76 & 35\end{array}$

Source: 1108012-02 Prepared: 10/07/11 Analyzed: 10/17/11

$\begin{array}{lcccccc}3.46 \mathrm{E} 4 & 2.62 \mathrm{E} 1 & \text { ug/g dry } & 4.26 \mathrm{E} 4 & 20.8 & 35 \\ 7.44 \mathrm{E} 2 & 9.65 \mathrm{E} 1 & " & 7.63 \mathrm{E} 2 & 2.57 & 35 \\ 4.36 \mathrm{E} 3 & 7.18 \mathrm{E} 0 & " & 3.97 \mathrm{E} 3 & 9.46 & 35 \\ 3.46 \mathrm{E} 2 & 7.10 \mathrm{E} 1 & " & 3.25 \mathrm{E} 2 & 6.28 & 35\end{array}$

Source: 1108012-02 Prepared \& Analyzed: 10/17/11

$\begin{array}{lcccccc}2.19 \mathrm{E} 5 & \mathrm{~N} / \mathrm{A} & \mathrm{ug} / \mathrm{L} & 5.00 \mathrm{E} 2 & 2.29 \mathrm{E} 5 & \mathrm{NR} & 75-125 \\ 5.33 \mathrm{E} 3 & \mathrm{~N} / \mathrm{A} & " & 1.25 \mathrm{E} 3 & 4.10 \mathrm{E} 3 & 98.6 & 75-125 \\ 2.12 \mathrm{E} 4 & \mathrm{~N} / \mathrm{A} & " & 5.00 \mathrm{E} 2 & 2.13 \mathrm{E} 4 & \mathrm{NR} & 75-125 \\ 2.23 \mathrm{E} 3 & \text { N/A } & " & 5.00 \mathrm{E} 2 & 1.74 \mathrm{E} 3 & 97.6 & 75-125\end{array}$




\section{Radionuclides by ICP-MS/1:1 Water Extract - Quality Control}

Environmental Science Laboratory

\begin{tabular}{|lrrrrrrrrrrrr}
\hline & & Reporting & & Spike & Source & & & $\%$ REC & & RPD \\
Analyte & Result & Limit & Units & Level & Result & $\%$ REC & Limits & RPD & Limit & Notes \\
\hline
\end{tabular}

\section{Batch 1J20001 - 1:1 Water Extract (ICP/ICPMS)}

Blank (1J20001-BLK1)

Technetium-99

Uranium 238

LCS (1J20001-BS1)

Technetium-99

Uranium 238

LCS (1J20001-BS2)

Technetium-99

Uranium 238

Duplicate (1J20001-DUP1)

Technetium-99

Uranium 238

Duplicate (1J20001-DUP2)

Technetium-99

Uranium 238

Post Spike (1J20001-PS1)

Technetium-99

Uranium 238

Batch 1J21002 - 1:1 Water Extract (ICP/ICPMS)

\section{Blank (1J21002-BLK1)}

Iodine-129

$\begin{array}{llc}<7.80 \mathrm{E}-5 & 7.80 \mathrm{E}-5 & \text { ug/g wet } \\ <1.60 \mathrm{E}-4 & 1.60 \mathrm{E}-4 & "\end{array}$

Prepared \& Analyzed: 10/20/11

Prepared \& Analyzed: 10/20/11

$\begin{array}{cccc}<3.90 \mathrm{E}-3 & 3.90 \mathrm{E}-3 & \text { ug/g wet } & 80-120 \\ <8.00 \mathrm{E}-3 & 8.00 \mathrm{E}-3 & " & 80-120\end{array}$

Prepared \& Analyzed: 10/20/11

$\begin{array}{cccc}<3.90 \mathrm{E}-3 & 3.90 \mathrm{E}-3 & \text { ug/g wet } & 80-120 \\ <8.00 \mathrm{E}-3 & 8.00 \mathrm{E}-3 & " & 80-120\end{array}$

Source: 1108012-01 Prepared \& Analyzed: 10/20/11

$\begin{array}{cccccc}<7.80 \mathrm{E}-5 & 7.80 \mathrm{E}-5 & \text { ug/g dry } & \text { ND } & 35 \\ 1.03 \mathrm{E}-3 & 1.60 \mathrm{E}-4 & \mu & 1.04 \mathrm{E}-3 & 0.297 & 35\end{array}$

Source: 1108012-02 Prepared \& Analyzed: 10/20/11

$\begin{array}{cccccc}<7.79 \mathrm{E}-5 & 7.79 \mathrm{E}-5 & \text { ug/g dry } & \text { ND } & & 35 \\ 1.06 \mathrm{E}-2 & 1.60 \mathrm{E}-4 & " & 9.40 \mathrm{E}-3 & 12.4 & 35\end{array}$

Source: 1108012-02 Prepared \& Analyzed: 10/20/11

$\begin{array}{lcccccc}1.06 \mathrm{E} 0 & \text { N/A } & \text { ug/L } & 1.09 \mathrm{E} 0 & 2.10 \mathrm{E}-5 & 97.5 & 75-125 \\ 1.92 \mathrm{E} 0 & \text { N/A } & " & 1.00 \mathrm{E} 0 & 9.40 \mathrm{E}-1 & 98.2 & 75-125\end{array}$




\section{Radionuclides by ICP-MS/1:1 Water Extract - Quality Control}

\section{Environmental Science Laboratory}

\begin{tabular}{|lrrrrrrrrrrr}
\hline & & Reporting & & Spike & Source & & & $\%$ REC & & RPD \\
Analyte & Result & Limit & Units & Level & Result & $\%$ REC & Limits & RPD & Limit & Notes \\
\hline
\end{tabular}

Batch 1J21002 - 1:1 Water Extract (ICP/ICPMS)

\begin{tabular}{|c|c|c|c|c|c|c|}
\hline Duplicate (1J21002-DUP1) & \multicolumn{3}{|c|}{ Source: 1108012-01 } & Prepared: 10/21/11 & \multirow[t]{2}{*}{ Analyzed: 10/25/11 } & \multirow[b]{2}{*}{35} \\
\hline Iodine-129 & $<7.44 \mathrm{E}-3$ & $7.44 \mathrm{E}-3$ & ug/g dry & ND & & \\
\hline Duplicate (1J21002-DUP2) & \multicolumn{3}{|c|}{ Source: $1108012-02$} & Prepared: 10/21/11 & Analyzed: 10/25/11 & \\
\hline Iodine-129 & $<7.43 \mathrm{E}-3$ & $7.43 \mathrm{E}-3$ & ug/g dry & ND & & 35 \\
\hline Post Spike (1J21002-PS1) & \multicolumn{3}{|c|}{ Source: $1108012-02$} & Prepared: 10/21/11 & Analyzed: 10/25/11 & \\
\hline Iodine-129 & $8.93 \mathrm{E}-1$ & N/A & $\mathrm{ug} / \mathrm{L}$ & $1.00 \mathrm{E} 0$ & $75-125$ & \\
\hline
\end{tabular}


RCRA Metals By PNNL-AGG-415/1:1 Water Extract - Quality Control

Environmental Science Laboratory

\begin{tabular}{|lrrrrrrrrrrr}
\hline & & Reporting & & Spike & Source & & & $\%$ REC & & RPD & \\
Analyte & Result & Limit & Units & Level & Result & $\%$ REC & Limits & RPD & Limit & Notes \\
\hline
\end{tabular}

Batch 1J13002 - 1:1 Water Extract (ICP/ICPMS)

Blank (1J13002-BLK1)

Chromium

Arsenic

Selenium

Silver

Cadmium

Lead

Mercury

LCS (1J13002-BS1)

Chromium

Arsenic

Selenium

Silver

Cadmium

Lead

Duplicate (1J13002-DUP1)

Chromium

Arsenic

Selenium

Silver

Cadmium

Lead

Mercury

Duplicate (1J13002-DUP2)

Chromium

Arsenic

Selenium

Silver

Cadmium

Lead

Mercury

$<6.8$

$\begin{array}{llc}<6.89 \mathrm{E}-3 & 6.89 \mathrm{E}-3 & \text { ug } / \text { g wet } \\ <5.67 \mathrm{E}-3 & 5.67 \mathrm{E}-3 & " \\ <1.58 \mathrm{E}-2 & 1.58 \mathrm{E}-2 & " \\ <6.26 \mathrm{E}-3 & 6.26 \mathrm{E}-3 & " \\ <7.71 \mathrm{E}-4 & 7.71 \mathrm{E}-4 & " \\ <2.71 \mathrm{E}-3 & 2.71 \mathrm{E}-3 & " \\ <1.46 \mathrm{E}-3 & 1.46 \mathrm{E}-3\end{array}$

Prepared \& Analyzed: 10/13/11

Prepared \& Analyzed: 10/13/11

\begin{tabular}{llcccc} 
& \multicolumn{5}{c}{ Prepared \& Analyzed. $10 / 13 / 11$} \\
$4.00 \mathrm{E} 0$ & $3.44 \mathrm{E}-1$ & ug/g wet & $5.00 \mathrm{E} 0$ & 99.9 & $80-120$ \\
$4.73 \mathrm{E} 0$ & $2.84 \mathrm{E}-1$ & $"$ & $5.00 \mathrm{E} 0$ & 94.6 & $80-120$ \\
$4.84 \mathrm{E} 0$ & $7.92 \mathrm{E}-1$ & $"$ & $5.00 \mathrm{E} 0$ & 96.7 & $80-120$ \\
$4.91 \mathrm{E} 0$ & $3.13 \mathrm{E}-1$ & $"$ & $5.00 \mathrm{E} 0$ & 98.1 & $80-120$ \\
$4.83 \mathrm{E} 0$ & $3.86 \mathrm{E}-2$ & $"$ & $5.00 \mathrm{E} 0$ & 96.6 & $80-120$ \\
$4.79 \mathrm{E} 0$ & $1.36 \mathrm{E}-1$ & $"$ & $5.00 \mathrm{E} 0$ & 95.7 & $80-120$
\end{tabular}

Source: 1108012-01 Prepared \& Analyzed: 10/13/11

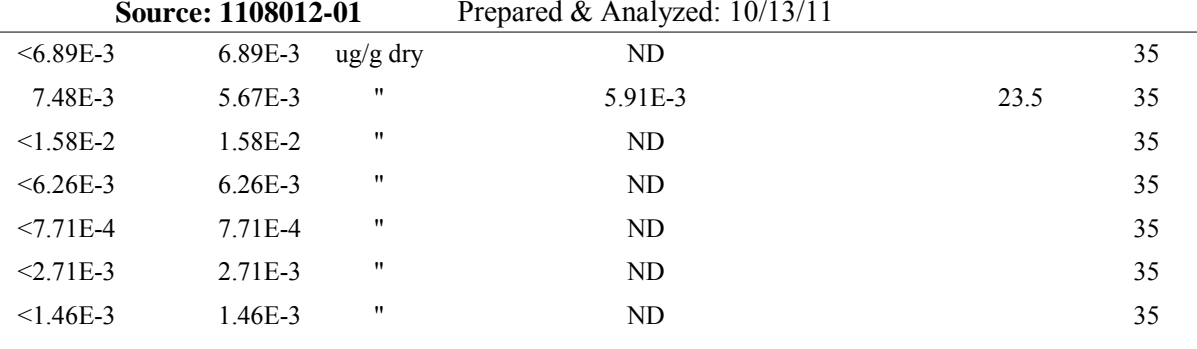

Source: 1108012-02 Prepared \& Analyzed: 10/13/11

\begin{tabular}{rccccc}
\multicolumn{7}{c}{ Source: 1108012-02 } & Prepared \& Analyzed: $10 / 13 / 11$ & \\
$<6.88 \mathrm{E}-3$ & $6.88 \mathrm{E}-3$ & ug/g dry & ND & 9.87 & 35 \\
$1.04 \mathrm{E}-2$ & $5.67 \mathrm{E}-3$ & $"$ & $9.46 \mathrm{E}-3$ & 35 \\
$<1.58 \mathrm{E}-2$ & $1.58 \mathrm{E}-2$ & $"$ & ND & & 35 \\
$<6.25 \mathrm{E}-3$ & $6.25 \mathrm{E}-3$ & $"$ & ND & ND & 35 \\
$<7.70 \mathrm{E}-4$ & $7.70 \mathrm{E}-4$ & $"$ & ND & 35 \\
$<2.71 \mathrm{E}-3$ & $2.71 \mathrm{E}-3$ & $"$ & ND & 35 \\
$<1.46 \mathrm{E}-3$ & $1.46 \mathrm{E}-3$ & $"$ & &
\end{tabular}


RCRA Metals By PNNL-AGG-415/1:1 Water Extract - Quality Control

Environmental Science Laboratory

\begin{tabular}{|c|c|c|c|c|c|c|c|c|c|c|}
\hline & & oorting & & Spike & Source & & $\%$ REC & & RPD & \\
\hline Analyte & Result & Limit & Units & Level & Result & $\%$ REC & Limits & RPD & Limit & Notes \\
\hline
\end{tabular}

Batch 1J13002 - 1:1 Water Extract (ICP/ICPMS)

Post Spike (1J13002-PS1)

Chromium

Arsenic

Selenium

Silver

Cadmium

Lead

Mercury
Source: 1108012-02

$5.08 \mathrm{E} 0$

$5.55 \mathrm{E} 0$

$5.12 \mathrm{E} 0$

$4.79 \mathrm{E} 0$

$4.86 \mathrm{E} 0$

$4.56 \mathrm{E} 0$

4.99E0
Prepared \& Analyzed: 10/13/11

$\begin{array}{cccccc}\text { N/A } & \text { ug/L } & 5.00 \mathrm{E} 0 & 3.68 \mathrm{E}-2 & 101 & 75-125 \\ \text { N/A } & " & 5.00 \mathrm{E} 0 & 9.46 \mathrm{E}-1 & 92.2 & 75-125 \\ \text { N/A } & " & 5.00 \mathrm{E} 0 & 2.67 \mathrm{E}-1 & 97 & 75-125 \\ \text { N/A } & " & 5.00 \mathrm{E} 0 & \text { ND } & 95.9 & 75-125 \\ \text { N/A } & " & 5.00 \mathrm{E} 0 & 7.97 \mathrm{E}-3 & 97 & 75-125 \\ \text { N/A } & " & 5.00 \mathrm{E} 0 & 3.74 \mathrm{E}-4 & 91.2 & 75-125 \\ \text { N/A } & " & 5.00 \mathrm{E} 0 & 1.20 \mathrm{E}-2 & 99.5 & 75-125\end{array}$




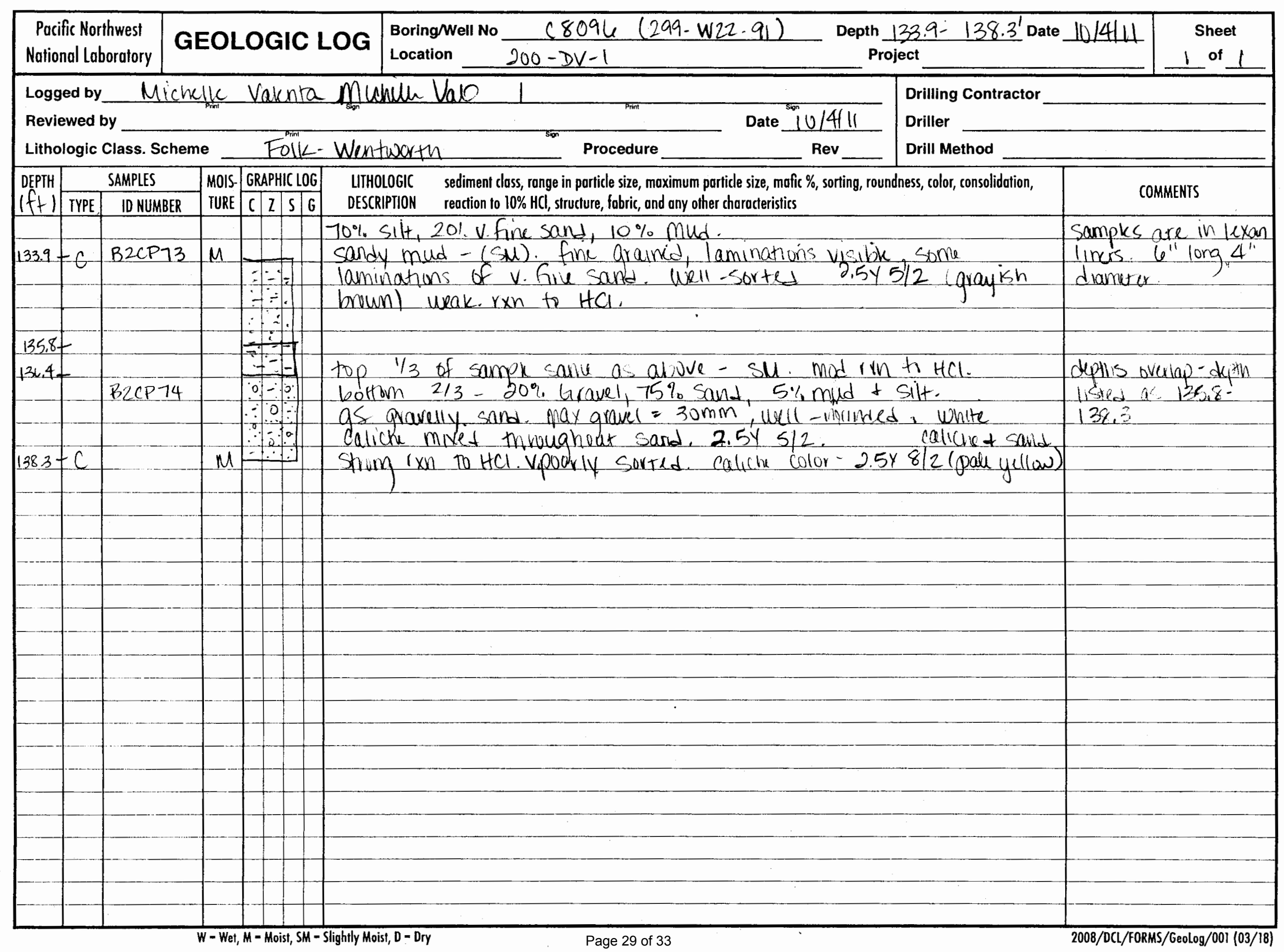



MILAMETERS

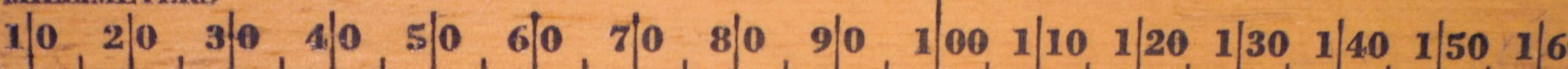

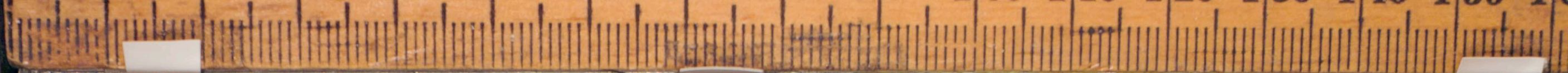

$\frac{\infty}{0}$

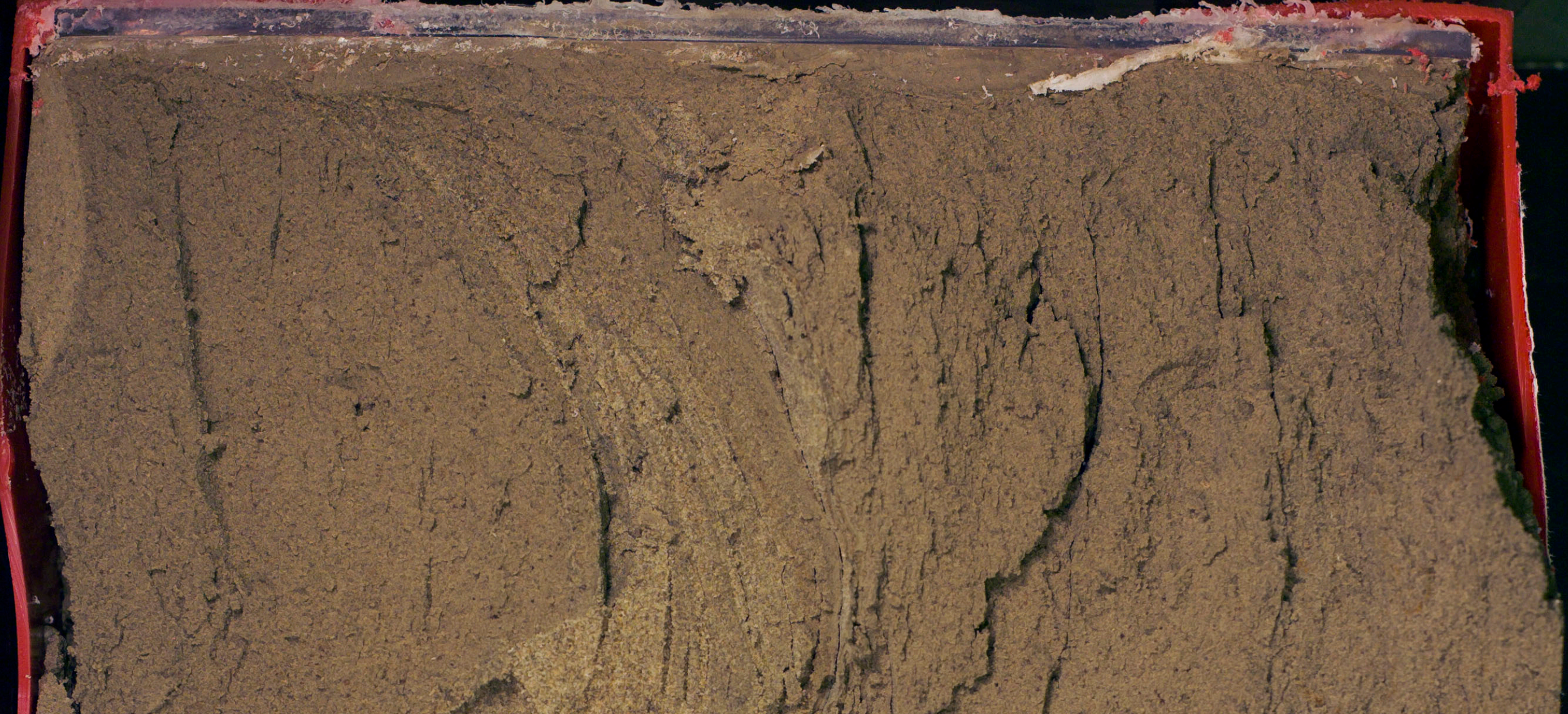

$\omega$
$\omega$
$\omega$
$\omega$
+
+
+
+

on

G

$\frac{\rho}{\rho}$
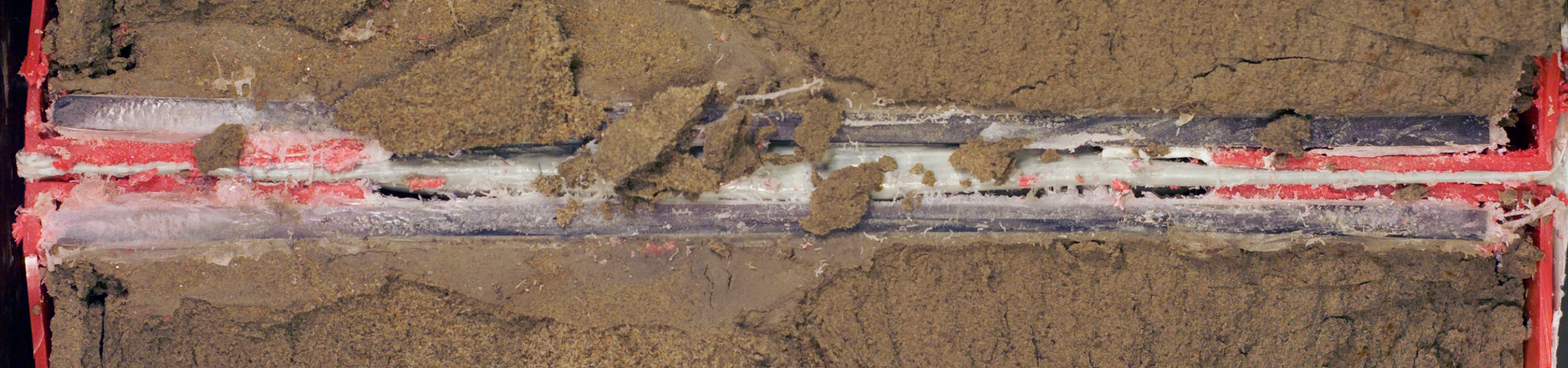

9.

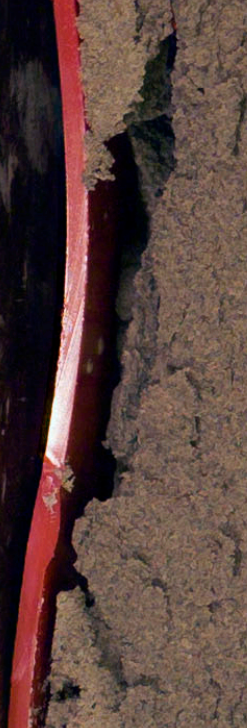




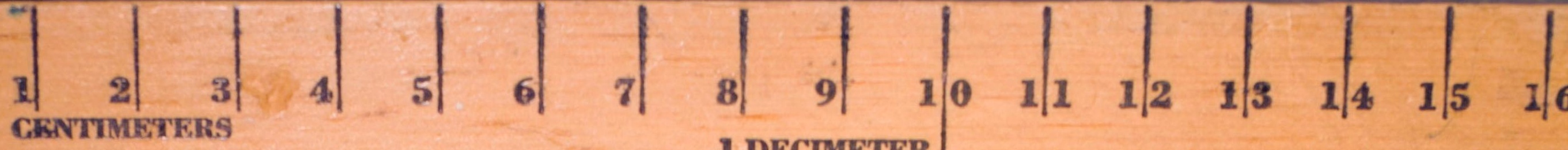
MHUIMETERS

1 DECIMETER

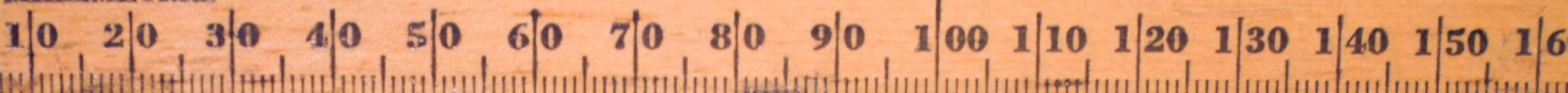

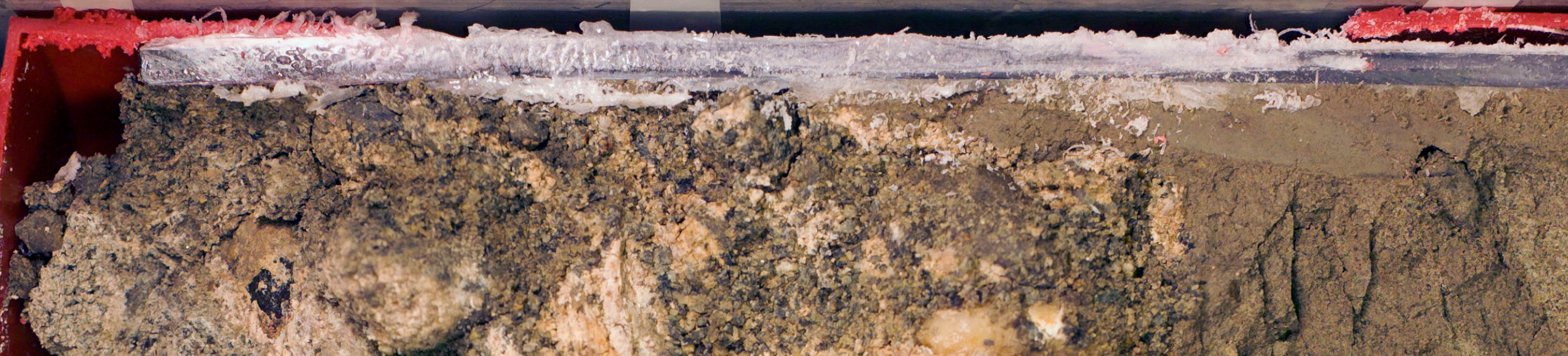

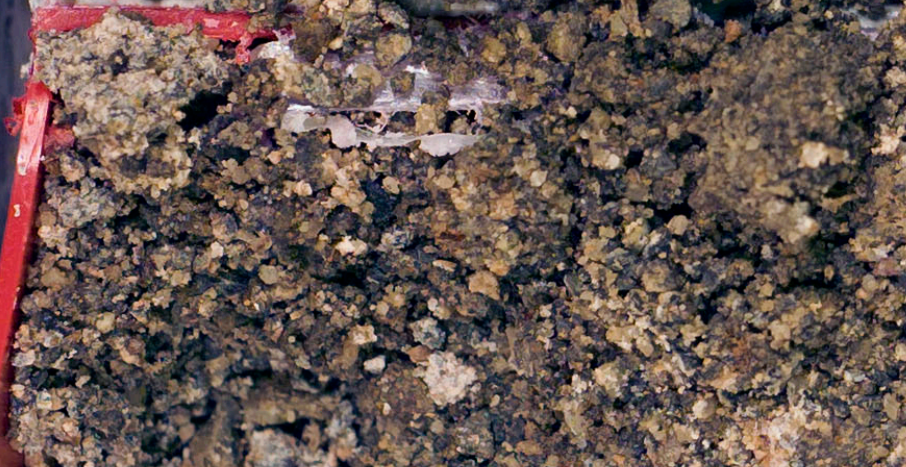

\section{1.t.}

terst?

zinge

ches

3

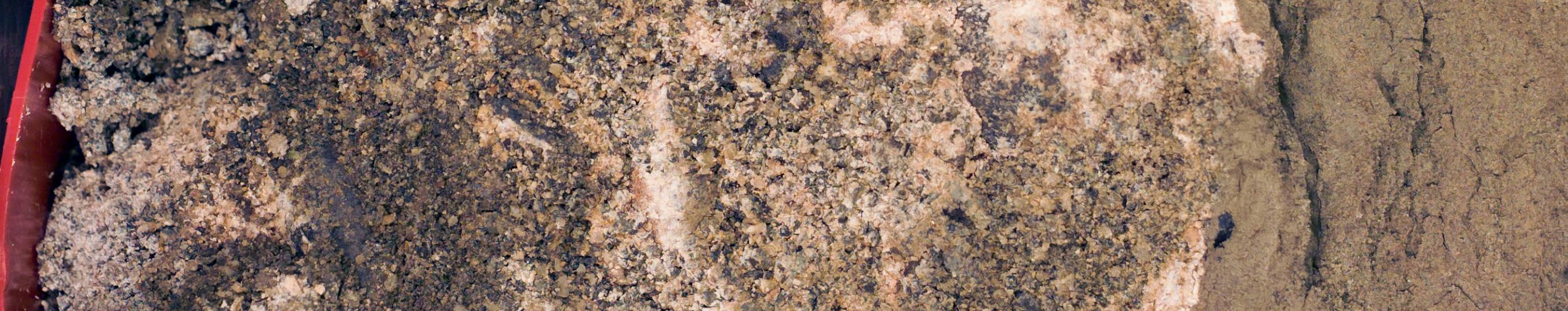

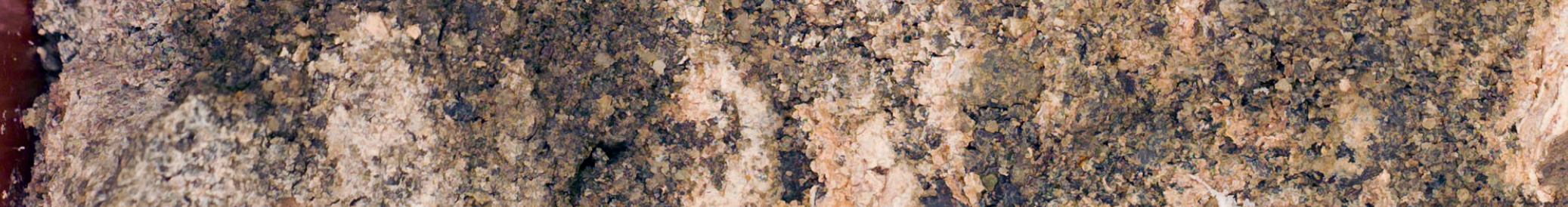

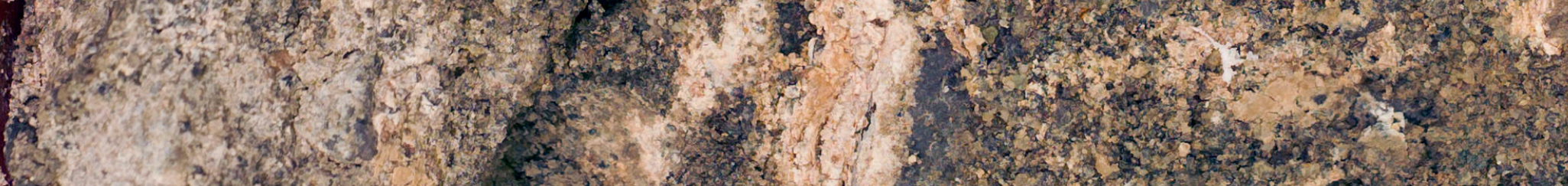




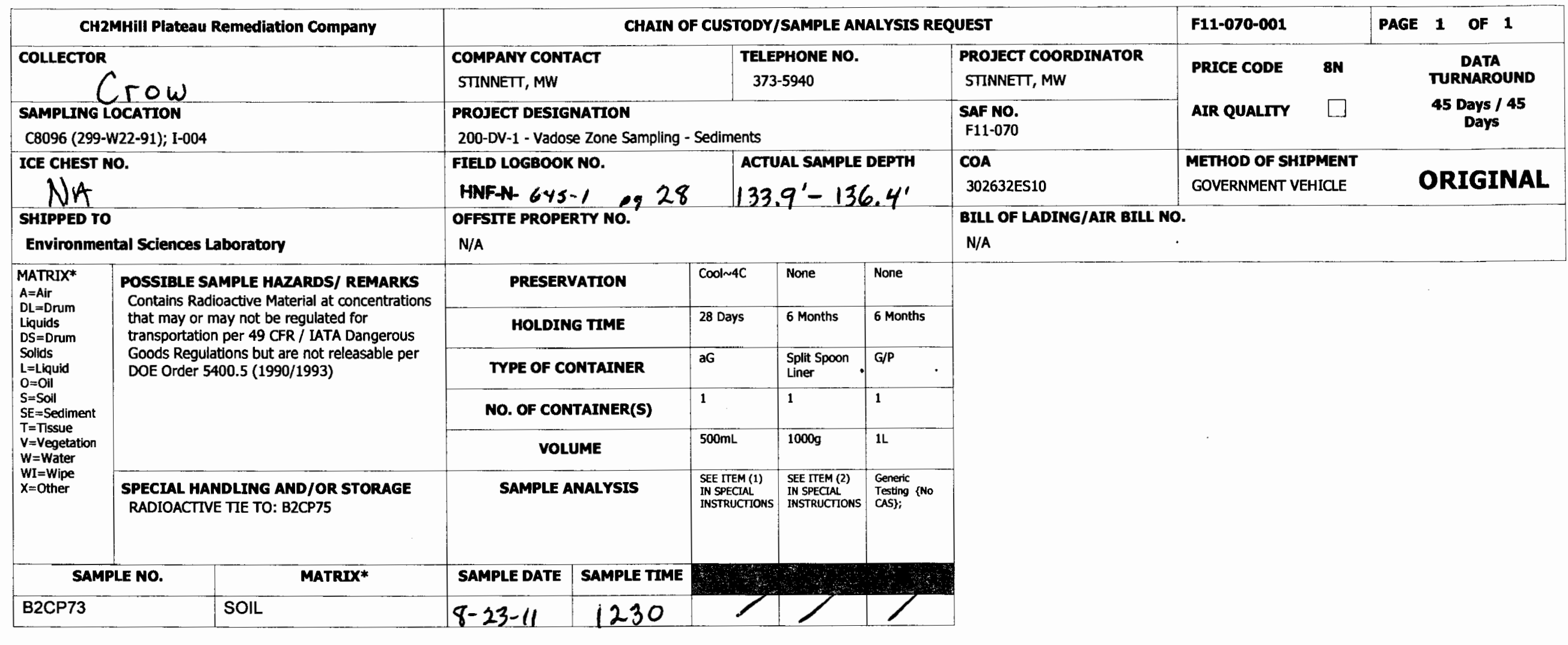

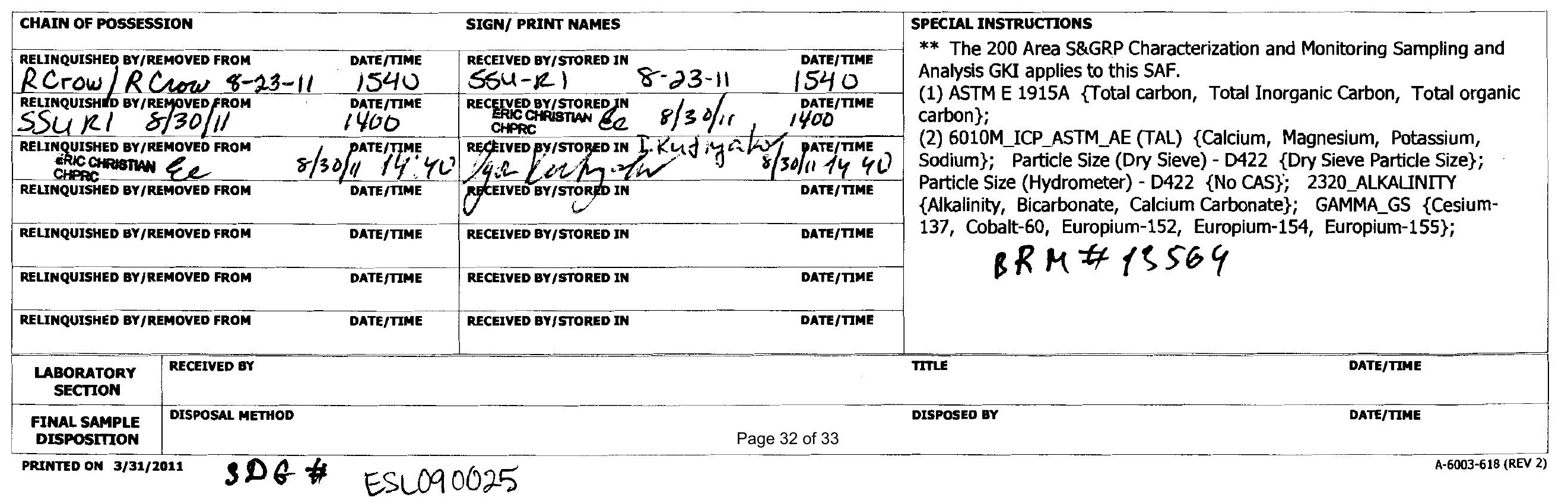




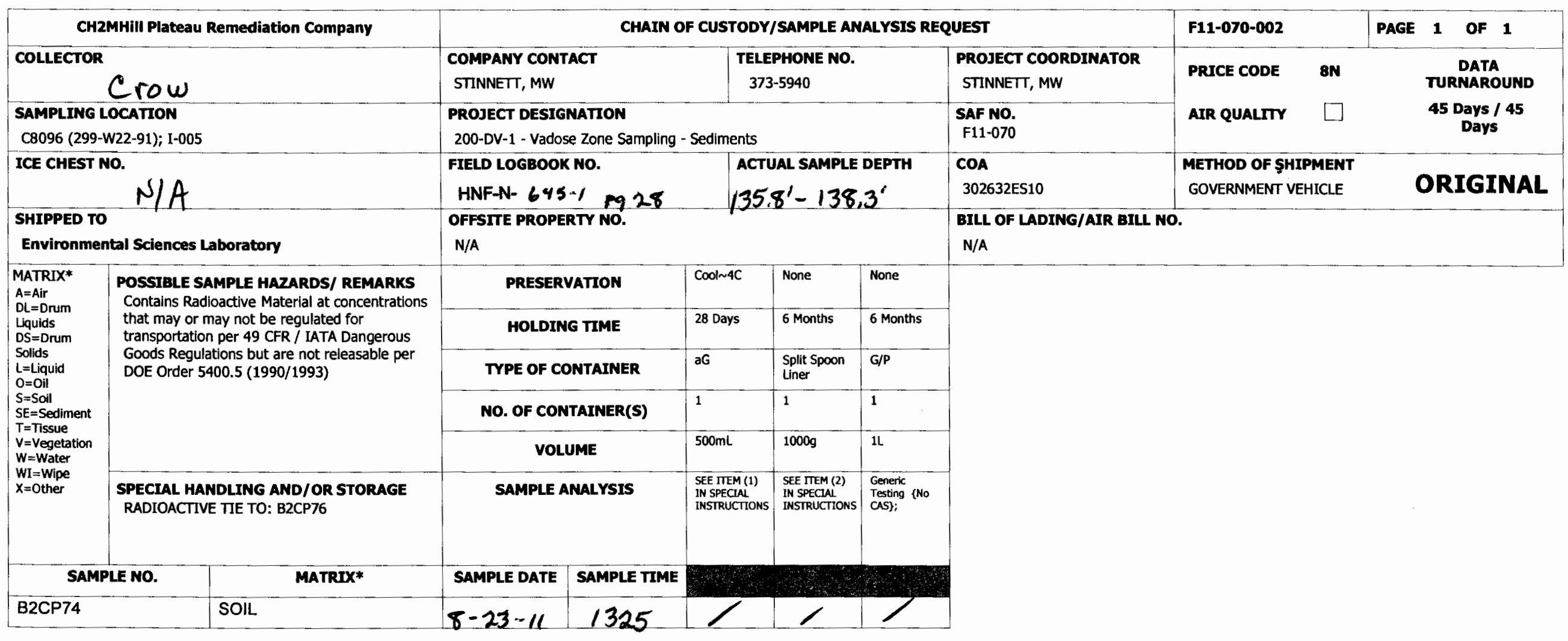

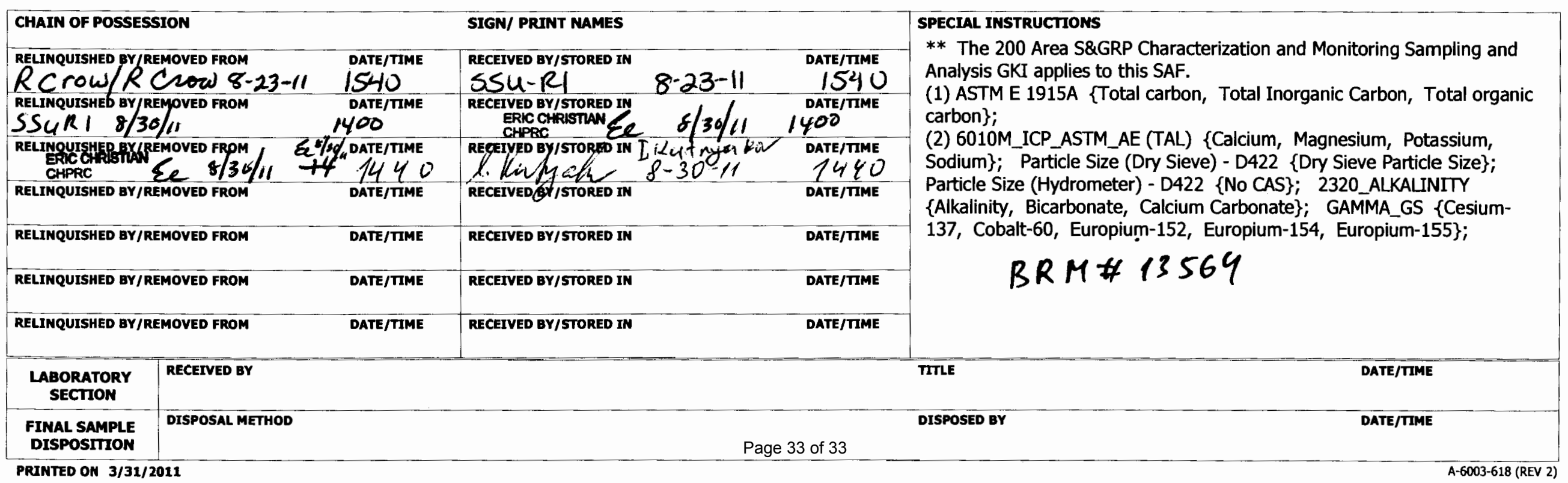

